\title{
White light-emitting contrast image capsule endoscopy for visualization of small intestine lesions: a pilot study
}

\section{다 (우우}

\begin{abstract}
Authors Miyachi', Toshiyuki Baba', Fumio Ishida', Shin-ei Kudo ${ }^{1}$

Institutions

1 Digestive Disease Center, Showa University Northern Yokohama Hospital, Yokohama, Kanagawa, Japan

2 Department of Endoscopy, Tokyo Medical and Dental University Hospital, Tokyo, Japan
\end{abstract}

Noriyuki Ogata ${ }^{1}$, Kazuo Ohtsuka ${ }^{1}$, , Seiko Sasanuma ${ }^{1}$, Masataka Ogawa ${ }^{1}$, Yasuharu Maeda ${ }^{1}$, Katsuro Ichimasa ${ }^{1}$, Yuichi Mori ${ }^{1}$, Masashi Misawa ${ }^{1}$, Toyoki Kudo ${ }^{1}$, Tomokazu Hisayuki ${ }^{1}$, Takemasa Hayashi ${ }^{1}$, Kunihiko Wakamura ${ }^{1}$, Hideyuki

submitted 14.6.2017

accepted after revision 3.1.2018

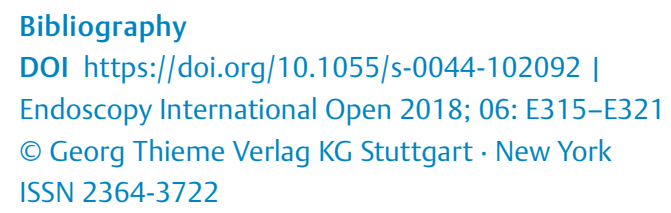

Corresponding author

Noriyuki Ogata, MD, Digestive Disease Center, Showa University, Northern Yokohama Hospital, Yokohama, Kanagawa 2248503, Japan

Phone: +81-45-949-7000

Fax: $+81-45-949-7263$

n.ogata@med.showa-u.ac.jp

\section{ABSTRACT}

Background and study aims Capsule endoscopy (CE) has become a routine means of diagnosing obscure gastrointestinal bleeding (OGIB) in the small intestine. Capsules using novel blue-enhanced white light-emitting diodes are expected to acquire clearer contrast images ( $\mathrm{Cls}$ ) of the small bowel vasculature. We conducted a pilot study to examine whether Cls facilitate visualization of small bowel erosions, ulcers, and areas of angioectasia compared with standard white light images (WLIs).

Patients and methods A total of 24 patients with OGIB were recruited in this study. The main outcome measure was visibility of lesions on Cls compared with WLIs. We also examined the color difference between lesions and normal mucosa $(\Delta \mathrm{E})$ with each imaging modality.

Results Three experienced physicians retrospectively evaluated 138 images of small bowel lesions (107 erosions, or ulcers, and 31 areas of angioectasia) obtained from 24CE examinations. The endoscopists judged that compared with WLIs, Cls afforded easier identification of erosions or ulcers in 29 of 107 cases $(27.1 \%)$, were non-inferior in 68 of 107 cases $(63.6 \%)$, and were inferior in 10 of 107 cases (9.3\%). Identification of angioectasia was judged to be easier with Cls in 15 of 31 cases (48.4\%), non-inferior in 13 of 31 cases $(41.9 \%)$, and inferior in 3 of 31 cases (9.7\%). $\Delta \mathrm{E}$ was significantly higher for Cls than WLIs, especially for angioectasia, potentially explaining why lesions were easier to visualize.

Conclusions Cls obtained by CE appear to facilitate identification of small bowel erosions, ulcers, and areas of angioectasia compared with WLIs.

\section{Introduction}

Image-enhanced endoscopy (IEE) improves detection, diagnosis, and treatment of gastrointestinal lesions [1,2]. IEE techniques are used to enhance views of the microvasculature and improve resolution of surface patterns and color differences. Several IEE modalities are in widespread clinical use, such as proprietary narrow band imaging (NBI, Olympus Corp., Tokyo, Japan) and flexible spectral color enhancement (FICE, Fujifilm Corp., Tokyo, Japan).
Capsule endoscopy (CE) allows visualization of the mucosa throughout the entire small intestine [3]. Several studies have shown that CE is an effective means of detecting lesions in the small bowel, particularly sites of obscure gastrointestinal bleeding (OGIB) [4-7]. A previous study demonstrated the feasibility of performing CE using a FICE digital processing system within a capsule workstation (Given Imaging, Yoqneam, Israel) [8]. Other reports have suggested that FICE may improve visibility of small intestine lesions $[9,10]$. The contrast capsule is a novel image-enhanced capsule endoscope with blue-enhanced white light-emitting diodes (WL-LEDs) that allow for acquisition 
of high-contrast images by selecting predominantly blue and green light wavelengths. The aim of this study was to assess whether high-contrast images captured by this contrast capsule improve the visibility of small intestine lesions compared with standard white light images (WLIs).

\section{Patients and methods}

\section{Patients and informed consent}

The study was performed from October 2010 to September 2012 at Showa University Northern Yokohama Hospital. A total of 24 patients with OGIB who were determined to be acceptable state for CE based on their history and physical examination findings were enrolled in this study. Exclusion criteria were as follows: a history of intestinal surgery, suspected intestinal obstruction, suspected inflammatory bowel disease, pregnancy, and age $<18$ years. All patients had recently undergone two endoscopic examinations (gastroscopy and colonoscopy) that showed negative findings. The conduct of the study was approved by the Ethics Committee of Showa University Northern Yokohama Hospital. All 24 patients provided written in-

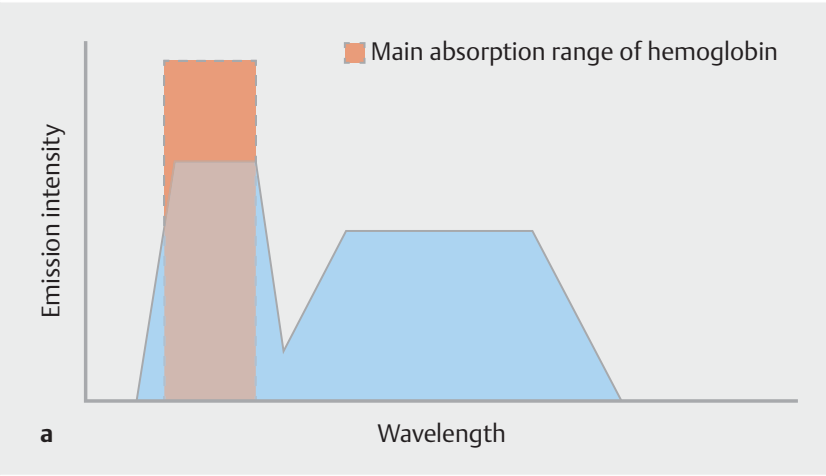

formed consent for use of their data. The study was undertaken in accordance with the Declaration of Helsinki.

\section{Contrast capsule}

The contrast capsule is an image-enhanced capsule endoscope. This is the conventional capsule (EC type1, Olympus Corp.) equipped with a special WL-LED. The WL-LED was selected to give increased illumination intensity in the blue wavelength range, which is appropriate for visualization of hemoglobin ( $\triangleright$ Fig.1a). WLIs are generated by using all red, green, and blue data obtained from a charge-coupled device in a conventional manner, whereas contrast images ( $\mathrm{Cls}$ ) are generated by

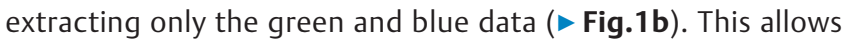
for greater differentiation between hemoglobin-rich tissue and less vascular structures, potentially contributing to better identification of bleeding points. The contrast capsule is an opticaldigital method analogous to NBI in the Tajiri and Niwa endoscopic imaging classification [11].

\section{Capsule endoscopy procedure}

The Olympus WS-1 software program (Olympus Corp.) was used to review, refine and quantify the images. Bowel preparation was with $500 \mathrm{~mL}$ of polyethylene glycol (PEG) and $20 \mathrm{~mL}$ of dimethicone; patients were instructed to swallow the capsule with the dimethicone solution after an overnight fast. Once the capsule had reached the duodenum as established by the real-time viewer [12], patients were asked to drink the PEG. Ingesting a small amount of PEG after swallowing the capsule significantly improves both CE image quality and cecal completion rate $[13,14]$. Three experienced endoscopists, each of whom had read more than $50 \mathrm{CE}$ videos, interpreted the $\mathrm{CE}$ images and a consensus interpretation was reached. If discre-

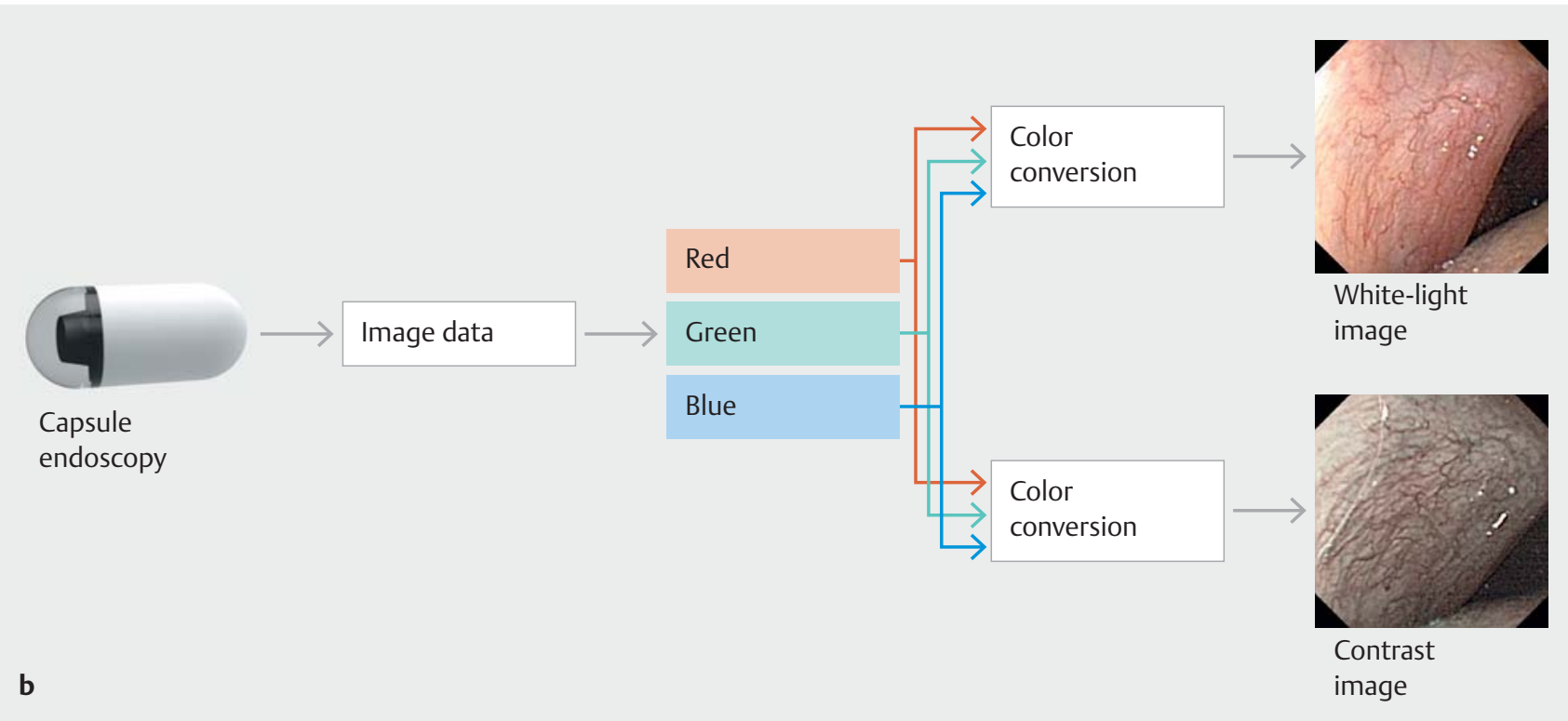

- Fig. 1 a The capsule endoscope was equipped with selected white light-emitting diodes, which provided increased illumination intensity in the blue light spectrum (the main absorption range of hemoglobin). b Contrast capsule endoscopy system configured predominantly with blue light and green light using the color illumination system. 


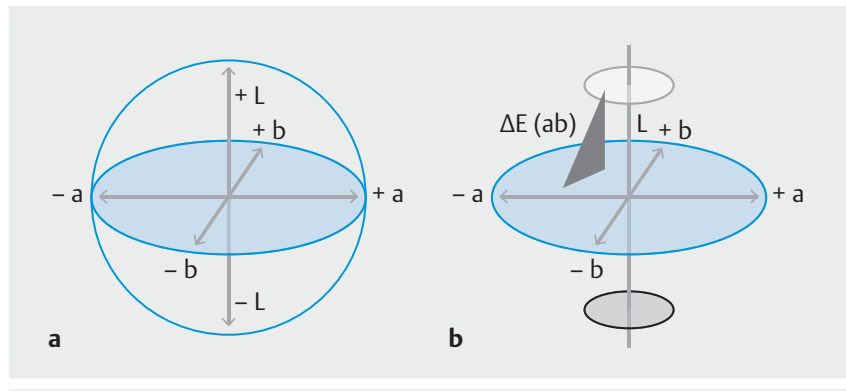

- Fig. 2 a Lab color space as defined by the International Commission on Illumination (CIE). $\mathbf{b}$ Color difference $(\Delta \mathrm{E})$ was calculated using the following equation: $\Delta \mathrm{E}=\left(\Delta \mathrm{L}^{2}+\Delta \mathrm{a}^{2}+\Delta \mathrm{b}^{2}\right)^{1 / 2}$ where $L$ represents lightness, a the red-green component and b the yellow-blue component.

pancies were observed, a consensus was reached after the findings were reviewed simultaneously by all endoscopists.

\section{Evaluation of contrast images}

Data on lesions in this study were managed by N. O., who was not involved in further evaluation of the images. The WLI and $\mathrm{Cl}$ of each lesion were displayed side by side. All lesions, including ulcerations/erosions and the area of angioectasia, were randomized. Three physicians (M. O., K.I., and K. O.) retrospectively compared the Cls with the corresponding WLIs. The physicians scored the Cls for visibility of small bowel lesions according to the following scale: 1 (improved visibility), 0 (visibility equivalent to that of WLI visibility), and -1 (decreased visibility). The three physicians' scores for each lesion were tallied. If an image earned a total score $\geq 2$, the image was considered im- proved; a score of 1 to -1 points indicated no change; and a score of $\leq-2$ indicated decreased visibility. We also examined interobserver and intraobserver agreement in evaluation of the Cls.

\section{Color contrast}

The color contrast of normal background mucosa and the lesions detected (erosions, ulcerations or areas of angioectasia) were compared by calculating the color difference $(\Delta \mathrm{E})$ for the WLIs and Cls. We used International Commission on Illumination (CIE) Lab to quantify the color and evaluate $\Delta \mathrm{E}$ ( $>$ Fig. 2) [15]. CIELab is a three-dimensional color space consisting of a black-white axis (L), a red-green axis (a) and a yellow-blue axis (b), where $L$ is lightness, $a$ is the red-green component, and $b$ is the yellow-blue component. We used CIELab to compare two points of interest and calculate the color difference ( $\triangleright$ Fig.3). Points of interest were selected manually by N.O., although the calculation of the color difference was done automatically.

\section{Detection rate}

To evaluate the detection rate of the small intestine lesions (erosions, ulcerations or areas of angioectasia), CE videos of 24 cases were evaluated by two physicians (S. S. and Y.M.) who had similar experience with endoscopy; one evaluated video $\mathrm{Cls}$ and the other evaluated video WLIs in a blinded fashion. Numbers of lesions detected by Cls and WLIs were compared.

\section{Statistical analysis}

The paired Student's $t$-test or the Wilcoxon signed-rank test was used to compare quantitative variables. Differences with $P$ values $<0.05$ were considered statistically significant. Interob-

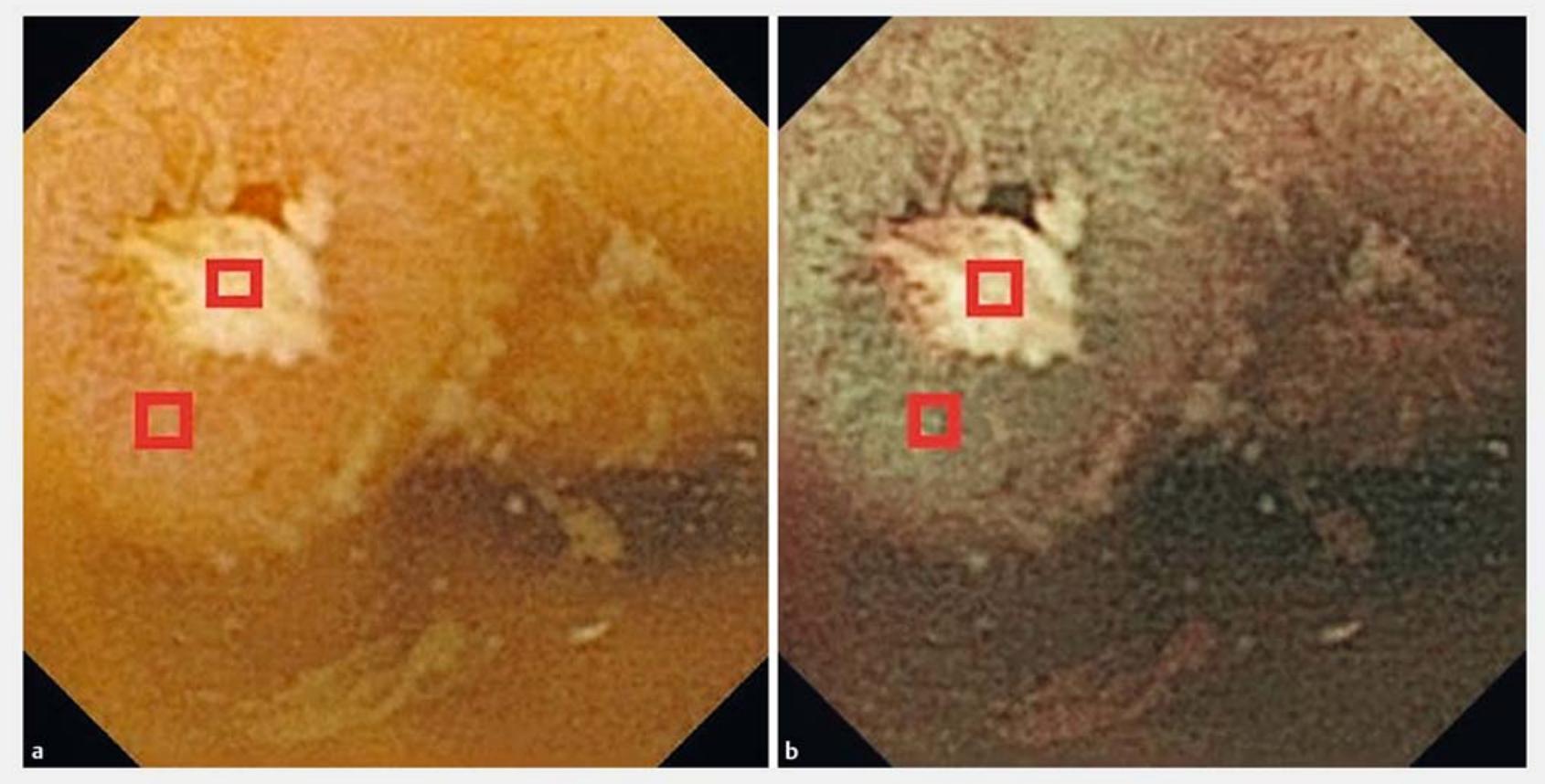

- Fig. 3 Color difference $(\Delta \mathrm{E})$ was compared between the detected lesion and the background normal mucosa. 
- Table 1 Characteristics of study participants.

\begin{tabular}{|l|c|}
\hline Profiles of enrolled patients & \\
\hline Total number of patients & 24 \\
\hline Sex M/F & $10 / 14$ \\
\hline Mean age (years) & 63.9 \\
\hline Overt ongoing bleeding & 1 \\
\hline Overt previous bleeding & 18 \\
\hline Occult bleeding & 5 \\
\hline NSAIDs & 8 \\
\hline Hospitalization & 10 \\
\hline M, male; F, female; NSAIDs, nonsteroidal anti-inflammatory drugs. \\
\hline
\end{tabular}

server and intraobserver agreement was quantified using the kappa statistic. Data are presented as the mean \pm standard deviation unless otherwise stated. Statistical analyses were performed with SPSS for Windows software version 15.0 (SPSS Inc., Chicago, IL, USA).

\section{Results}

A total of 24 patients were recruited for this study. The characteristics of the patients and the indications for CE are shown in - Table 1. Small intestine lesions were detected in 15 patients (62.5\%), including 107 erosions or ulcers and 31 areas of angioectasia. Small intestinal ulcerations/erosions were identified in 6 of 8 patients taking nonsteroidal anti-inflammatory drugs (NSAIDs). Consequently, 138 lesions were used for this validation study. The completion rate was $75.0 \%$ [84.6\% (11/14) for outpatients and $70.0 \%$ (7/10) for hospitalized patients]. The mean small bowel transit time was $250.1 \pm 114.9 \mathrm{~min}$. The 138 lesions in which the final diagnoses were confirmed by several modalities including CE, single-balloon enteroscopy, a medical history, and follow-up observation were chosen on the basis of their overall acceptability on CE examination by three experienced endoscopists (Y.M., M. M., and H.M.) who had extensive experience with $C E$.

\section{Lesion visibility}

For erosions or ulcers, Cls were considered to afford better visibility in $27.1 \%$ of cases (29 of 107 ), equivalent visibility in $63.6 \%$ of cases (68 of 107 ), and inferior visibility in $9.3 \%$ of cases (10 of 107) $(P=0.024)$. The interobserver and intraobserver kappa values for visibility were 0.45 and 0.57 , respectively. For areas of angioectasia, Cls were considered to afford better visibility in $48.4 \%$ of cases ( 15 of 31 ), equivalent visibility in $41.9 \%$ of cases ( 13 of 31 ), and inferior visibility in $9.7 \%$ of cases (3 of 31) $(P=0.047)$ ( $\vee$ Table 2$)$. The interobserver and intraobserver kappa values for lesion visibility were 0.49 and 0.58 , respectively.

\section{Color difference}

For erosions and ulcers, $\Delta \mathrm{E}$ for $\mathrm{Cl}$ s was significantly higher than WLIs $(31.9 \pm 12.4$ compared with $18.7 \pm 9.7$, respectively; $P<$ $0.001)$. This was also the case for angioectasia $(38.4 \pm 15.5$, compared with $27.1 \pm 13.6$, respectively; $P=0.003)(\triangleright$ Table 3$)$.

\section{Detection rate}

Detection of lesions with Cls and WLIs according to lesion type is shown in $>$ Table 4. Of a total of 107 erosions or ulcers, 98 were identified by $\mathrm{Cls}$ and 72 were detected by WLIs $(P<$ $0.001)$. Of a total of 31 areas of angioectasia, 28 were identified by $\mathrm{Cls}$ and 20 were detected by WLIs $(P=0.015)$.

\section{Discussion}

The current study is the first to assess the clinical utility of $\mathrm{Cls}$ for detection of small intestine lesions in patients with OGIB undergoing CE. Our findings suggest that Cls obtained by CE may improve visibility of small bowel erosions, ulcers, and areas of angioectasia compared with WLIs. The $\Delta \mathrm{E}$ between lesions and the background normal mucosa was significantly higher with $\mathrm{Cls}$ than WLIs. The high $\Delta \mathrm{E}$ that we observed with Cls (particularly that in angioectasia) likely explains the improved visibility.

Clinical effectiveness of the novel contrast capsule that we used has already been reported $[16,17]$. Aihara et al. [16] performed a feasibility study that demonstrated imaging characteristics of the contrast capsule. In the current study, we evaluated the ability to identify small intestine erosions, ulcers, and area of angioectasia in Cls compared with WLIs. CE is a well-established modality for detection and diagnosis of OGIB. Many reports have assessed the diagnostic yield of CE in OGIB, but the sensitivity of the technique remains unknown. Computed virtual chromoendoscopy may improve the diagnostic yield in OGIB. The feasibility of performing CE using the FICE digital processing system within the capsule workstation has recently been demonstrated; FICE reportedly enhances lesions on CE images [8]. Several reports have described the efficacy of the FICE technique in CE $[8,9,18]$. Imagawa et al. [9] reported that

- Table 2 Evaluation of contrast images for visibility of lesions.

\begin{tabular}{|l|l|l|l|l|}
\hline & \multicolumn{2}{|c|}{ Number of cases (\%) } & & Total number of cases (\%) \\
\hline Lesion & Improved visibility & Equivalent visibility & Decreased visibility & Total \\
\hline Erosion/ulcer & $29(27.1)$ & $68(63.6)$ & $10(9.3)$ & $107(100)$ \\
\hline Angioectasia & $15(48.4)$ & $13(41.9)$ & $3(9.7)$ & $31(100)$ \\
\hline
\end{tabular}


- Table 3 Color difference between identified lesions and normal background mucosa.

\begin{tabular}{|l|l|l|l|}
\hline \multirow{2}{*}{ Lesion } & \multicolumn{2}{|l|}{ Color difference, $\Delta \mathbf{E}$} & \\
\hline & $\begin{array}{l}\text { Contrast } \\
\text { images }\end{array}$ & $\begin{array}{l}\text { White light } \\
\text { images }\end{array}$ & P value \\
\hline Erosion/ulcer & $31.9 \pm 12.4$ & $18.7 \pm 9.7$ & $<0.001$ \\
\hline Angioectasia & $38.4 \pm 15.5$ & $27.1 \pm 13.6$ & 0.003 \\
\hline
\end{tabular}
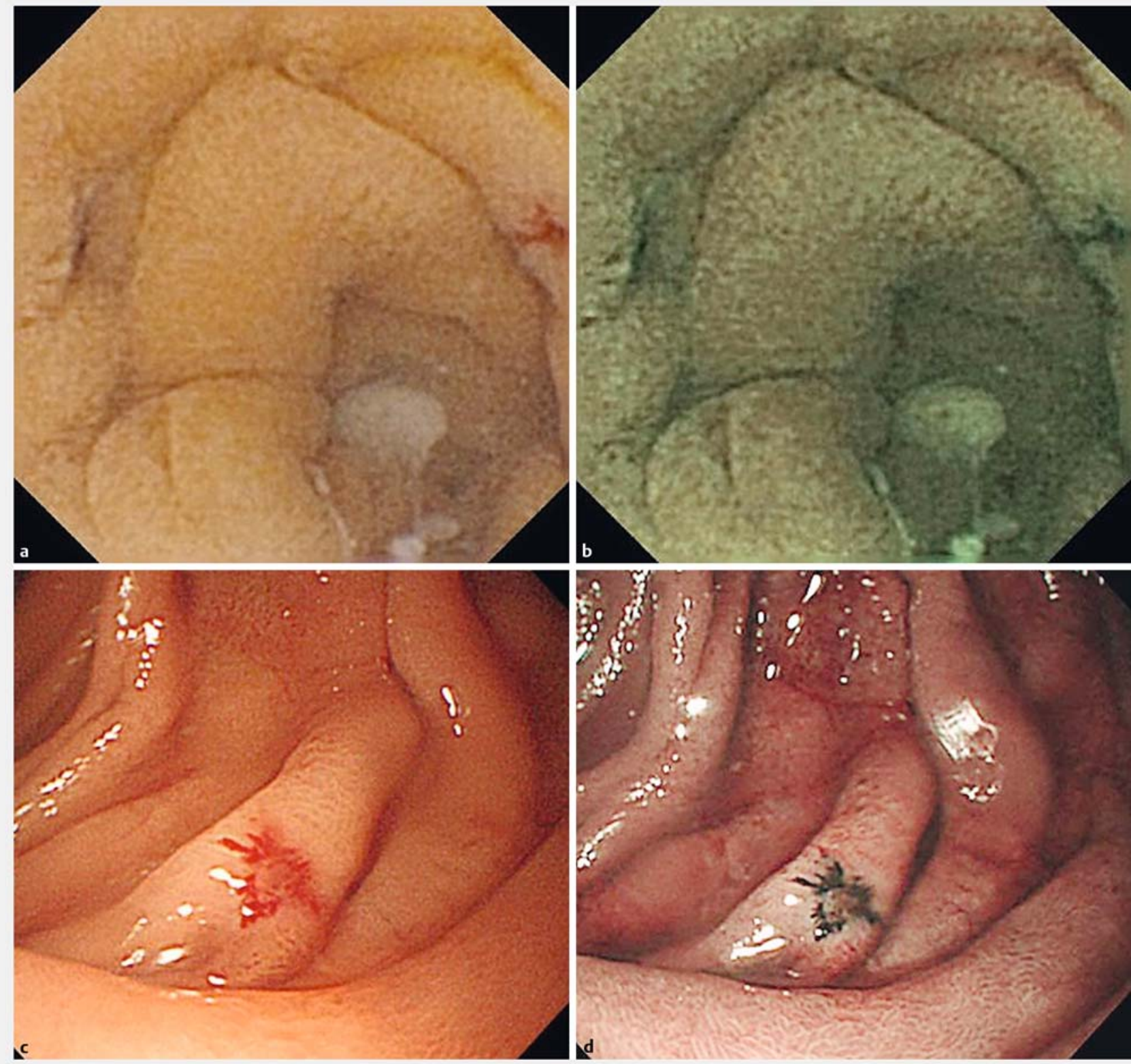

Fig. 4 Capsule endoscopy images of an area of small bowel angioectasia: a white light and $\mathbf{b}$ contrast images; c example of a contrast image and $\mathbf{d}$ narrow band image obtained by single-balloon endoscopy.

- Table 4 Number of lesions detected by contrast images and white light images.

\begin{tabular}{|l|l|l|l|}
\hline Lesion & $\begin{array}{l}\text { Contrast } \\
\text { images }\end{array}$ & $\begin{array}{l}\text { White light } \\
\text { images }\end{array}$ & P value \\
\hline Erosion/ulcer & 98 & 72 & $<0.001$ \\
\hline Angioectasia & 28 & 20 & 0.015 \\
\hline
\end{tabular}


FICE improved visibility of small bowel tumors, angioectasia, erosions and ulcers compared with WLIs. They also reported that FICE was a particularly useful means of improving image quality in areas of angioectasia, findings that are broadly comparable with ours. Employing an approach similar to that used in our study, Sato et al. [18] demonstrated the clinical utility of CE using FICE and CIELab methods to diagnose small bowel lesions. They demonstrated that CE with FICE enhanced the contrast between lesions and background mucosa, facilitating visual diagnosis of small bowel erosions, ulcers and angioectasia. However, Gupta et al. [19] reported that FICE did not significantly improve detection of small bowel lesions in comparison with WLIs [19].

The FICE technique facilitates CE by permitting selection of wavelengths and spectra appropriate for a variety of purposes (FICE1, 2, and 3). Nonetheless, contrast capsule technology fundamentally differs from FICE technology. In FICE, post-acquisition processing is performed on the images obtained from a conventional WL-LED, but images acquired using the contrast capsule technique are converted from a blue-enhanced WL-LED designed to reveal areas where hemoglobin is present. Consequently, Cls enhance blood vessels. The contrast capsule visualizes highly vascular lesions, such as angioectasia, in dark green with strong color contrast with normal mucosa. Such characteristics are broadly comparable with those of NBI ( $\triangleright$ Fig.4), which is an effective means of detecting cancers in the esophagus, stomach, and colon [20-22]. This characteristic of $\mathrm{Cl}$ explains the improvement in the detection rate of angioectasia in our study.

We also found that Cls improved the detection rate of erosions and ulcers compared with WLIs. A previous study showed that FICE improves the detection rate of small intestine lesions $[23,24]$, with FICE1 and FICE2 suited to the detection of erosions, ulcers, and angioectasia. Whether contrast capsule can further improve the ability to detect and diagnose small intestine lesions is not yet completely clear, but our findings suggest that it can produce clear images of vascular areas that would plausibly aid in diagnosis of erosions, ulcers, and angioectasia. Our current practice is to read capsule video images using WLI first and review Cls thereafter, but in the future, it might be possible to acquire contrast video images.

Our study had some limitations. First, its retrospective design may have resulted in selection bias. Second, it was a pilot study based in a single center with a relatively small number of patients. We did not assess the Cls to evaluate the lesion characteristics in this study. Such evaluations will be performed in the future.

\section{Conclusion}

In conclusion, $\mathrm{Cls}$ using $\mathrm{CE}$ increases $\Delta \mathrm{E}$ and consequently appears to improve the visibility of small intestine lesions. The technique could therefore be a useful diagnostic tool, particularly when seeking bleeding points in OGIB, but large prospective studies will be needed to illuminate its specificity, sensitivity, and clinical utility.

\section{Acknowledgements}

We are grateful to Olympus Corp.for providing the contrast capsule devices. The authors express great thanks to Chiaki Nishimura for providing advice regarding the statistical analysis and to all members of the Digestive Disease Center, Showa University Northern Yokohama Hospital for their excellent assistance. Finally, we thank Angela Morben, DVM, ELS, from Edanz Group (www.edanzediting.com/ac) for editing a draft of this manuscript.

\section{Competing interests}

None

References

[1] Pohl J, May A, Rabenstein T et al. Computed virtual chromoendoscopy: a new tool for enhancing tissue surface structures. Endoscopy 2007; 39: $80-83$

[2] Pohl J, May A, Rabenstein T et al. Comparison of computed virtual chromoendoscopy and conventional chromoendoscopy with acetic acid for detection of neoplasia in Barrett's esophagus. Endoscopy 2007; 39: 594- 598

[3] Iddan G, Meron G, Glukhovsky A et al. Wireless capsule endoscopy. Nature 2000; 405: 417

[4] Apostolopoulos P, Liatsos C, Gralnek IM et al. Evaluation of capsule endoscopy in active, mild-to-moderate, overt, obscure GI bleeding. Gastrointest Endosc 2007; 66: 1174 - 1181

[5] Pennazio M, Santucci R, Rondonotti E et al. Outcome of patients with obscure gastrointestinal bleeding after capsule endoscopy: Report of 100 consecutive cases. Gastroenterology 2004; 126: 643-653

[6] Triester SL, Leighton JA, Leontiadis Gl et al. A meta-analysis of the yield of capsule endoscopy compared to other diagnostic modalities in patients with obscure gastrointestinal bleeding. Am J Gastroenterol 2005; 100: $2407-2418$

[7] Raju GS, Gerson L, Das A et al. American Gastroenterological Association (AGA) Institute medical position statement on obscure gastrointestinal bleeding. Gastroenterology 2007; 133: 1694 - 1696

[8] Pohl J, Aschmoneit I, Schuhmann S et al. Computed image modification for enhancement of small-bowel surface structures at video capsule endoscopy. Endoscopy 2010; 42: 490-492

[9] Imagawa H, Oka S, Tanaka S et al. Improved visibility of lesions of the small intestine via capsule endoscopy with computed virtual chromoendoscopy. Gastrointest Endosc 2011; 73: 299-306

[10] Matsumura T, Arai M, Sato T et al. Efficacy of computed image modification of capsule endoscopy in patients with obscure gastrointestinal bleeding. World J Gastrointest Endosc 2012; 4: 421-428

[11] Tajiri H, Niwa H. Proposal for a consensus terminology in endoscopy: how should different endoscopic imaging techniques be grouped and defined? Endoscopy 2008; 40: 775-778

[12] Ogata $\mathrm{H}$, Kumai K, Imaeda $\mathrm{H}$ et al. Clinical impact of a newly developed capsule endoscope: usefulness of a real-time image viewer for gastric transit abnormality. J Gastroenterol 2008; 43: 186-192

[13] Endo H, Kondo Y, Inamori M et al. Ingesting $500 \mathrm{ml}$ of Polyethylene Glycol Solution During Capsule Endoscopy Improves the Image Quality and Completion Rate to the Cecum. Dig Dis Sci 2008; 53: 3201 3205 
[14] Hosono K, Endo H, Sakai E et al. Optimal Approach for Small Bowel Capsule Endoscopy Using Polyethylene Glycol and Metoclopramide with the Assistance of a Real-Time Viewer. Digestion 2011; 84: 119125

[15] Kuehni RG. Color-tolerance data and the tentative CIE $1976 \mathrm{~L}$ a b formula. J Opt Soc Am 1976; 66: 497 - 500

[16] Aihara H, Ikeda K, Tajiri H. Image-enhanced capsule endoscopy based on the diagnosis of vascularity when using a new type of capsule. Gastrointest Endosc 2011; 73: $1274-1279$

[17] Hatogai K, Hosoe N, Imaeda $\mathrm{H}$ et al. Role of enhanced visibility in evaluating polyposis syndromes using a newly developed contrast image capsule endoscope. Gut Liver 2012; 6: 218 - 222

[18] Sato Y, Sagawa T, Hirakawa M et al. Clinical utility of capsule endoscopy with flexible spectral imaging color enhancement for diagnosis of small bowel lesions. Endosc Int Open 2014; 2: E80 - 87

[19] Gupta T, Ibrahim M, Deviere J et al. Evaluation of Fujinon intelligent chromo endoscopy-assisted capsule endoscopy in patients with ob- scure gastroenterology bleeding. World J Gastroenterol 2011; 17 : $4590-4595$

[20] Kaltenbach T, Sano Y, Friedland S et al. American Gastroenterologica Association (AGA) Institute technology assessment on image-enhanced endoscopy. Gastroenterology 2008; 134: 327 - 340

[21] Kaltenbach T, Soetikno R. Image-enhanced endoscopy is critical in the detection, diagnosis, and treatment of non-polypoid colorectal neoplasms. Gastrointest Endosc Clin N Am 2010; 20: 471 - 485

[22] Gono K, Obi T, Yamaguchi M et al. Appearance of enhanced tissue features in narrow-band endoscopic imaging. J Biomed Opt 2004; 9: $568-577$

[23] Imagawa H, Oka S, Tanaka S et al. Improved detectability of smallbowel lesions via capsule endoscopy with computed virtual chromoendoscopy: a pilot study. Scand J Gastroenterol 2011; 46: 1133-1137

[24] Konishi M, Shibuya T, Mori H et al. Usefulness of flexible spectral imaging color enhancement for the detection and diagnosis of small intestinal lesions found by capsule endoscopy. Scand J Gastroenterol 2014; 49: 501 - 505 